\title{
A Formal Framework for Cognitive Models of Multitasking
}

\author{
Michael Lesnick ${ }^{1 * \uparrow}$, Sebastian Musslick ${ }^{2 *}$, Biswadip Dey ${ }^{3}$, Jonathan D. Cohen ${ }^{1,4}$ \\ ${ }^{2}$ Department of Mathematics and Statistics, University at Albany, Albany, NY 12222, USA. \\ ${ }^{2}$ Princeton Neuroscience Institute, Princeton University, Princeton, NJ 08544, USA. \\ ${ }^{3}$ Department of Mechanical and Aerospace Engineering, Princeton University, Princeton, NJ 08544, USA. \\ ${ }^{4}$ Department of Psychology, Princeton University, Princeton, NJ 08544, USA. \\ * These authors have contributed equally to this study. \\ *Corresponding Author: mlesnick@albany.edu
}

March 14, 2020

\begin{abstract}
This note introduces mathematical foundations for modeling of human multitask performance. Using basic definitions from set theory and graph theory, we introduce formal definitions of the environment in which multitasks are performed, of an agent which attempts to perform a multitask, and of the success rate of the agent on a multitask. Drawing on the recent literature on modeling of multitasking, we give two simple examples of multitasking agents, and illustrate the performance of these agents on two mutitasking problems: the well-known Stroop task, and a more complex variant.
\end{abstract}

Understanding how human cognitive architecture enables and constrains our ability to simultaneously perform multiple tasks is a fundamental problem in cognitive science. Recent work has approached this problem from a mathematical and computational perspective. While significant progress has been made (Townsend \& Wenger, 2004a; Feng, Schwemmer, Gershman, \& Cohen, 2014; Logan \& Gordon, 2001; Meyer \& Kieras, 1997; Musslick et al., 2016; Pashler, 1994; Welford, 1952; Wickens, 1991), the field has hitherto lacked a common formal language, making it difficult to relate different models of multitasking. The aim of this paper is to propose such a formal language, with examples, and to explain how this language unifies and makes precise ideas appearing in the recent work.

At a high level, the key features of the multitasking formalism presented here are abstract, formal definitions of each of the following:

1. The environment of multitasks that might be performed,

2. The agent which performs these tasks,

3. The success rate of an agent on a multitask.

Our definition of agent is intended as a simultaneous abstraction of human experimental participants, artificial neural networks (or other machine learning models of multitask performance), 
and other simpler models of multitask performance (see Section 4.1 for the discussion of one such model). Our definition of an agent has just enough structure to allow for a meaningful definition of the success rate of an agent.

We do not attempt here to fully model the many nuances of human multitasking; rather, we offer simple definitions at a high level of abstraction that we hope can serve as a starting point for more nuanced models. In particular, we do not explicitly model temporal dynamics, though these clearly are an essential part of the full story. It should be straightforward to enrich our models to accommodate temporal dynamics (e.g., learning rate and response time), and this is a subject for future work.

\section{Set Theory and Graph Theory Preliminaries}

Throughout the paper, we make extensive use of some basic definitions and notation from set theory and graph theory. In this section, we review these. Readers comfortable with the basic definitions of set theory and graph theory should feel free to skim or skip this section.

\subsection{Set Theory Preliminaries}

Sets. For our purposes, the following informal definition of a set will suffice:

Definition 1.1 (Set). A set is a collection of distinct elements.

Example 1.2. $S_{1}=\{A, B\}$ is a set. This is the set consisting of the two letters $A$ and $B$.

As shown above, we can specify a set by listing its elements. When we do that, we put the elements in curly braces. The order in which we write the elements in the curly braces doesn't matter.

Example 1.3. $S_{2}=\{0,1,2\}$ is a set, consisting of the three numbers 0,1 , and 2 .

Example 1.4. The real numbers form a set, usually denoted $\mathbb{R}$. Unlike the previous two examples, this set has infinitely many elements.

Example 1.5. \{\} is a set, called the empty set. This is the unique set with no elements.

Example 1.6. We can have a set whose elements are themselves sets. For example for sets $S_{1}, S_{2}$, and $S_{3}$ defined as above, $S_{4}=\left\{S_{1}, S_{2}\right\}$ is a set.

Example 1.7. A set with a single element is called a singleton set. For example, $\{2\}$ is a set with one element. Note that this is not the same as the number 2; rather it is the singleton set with element 2.

If a set $S$ contains an element $a$, we write $a \in S$. Otherwise, we write $a \notin S$.

Example 1.8. $A \in S_{1}$, but $A \notin S_{2}$.

We say sets $S$ and $T$ equal, and write $S=T$, if the elements of $S$ and $T$ are the exactly same. If $S$ and $T$ are not equal, we write $S \neq T$.

Sometimes it will be convenient for us to specify an order of the elements in a finite set $S$. When such an order is given, we say that $S$ is ordered.

Definition 1.9 (Function). Here is the usual (informal) definition of a function: Given sets $S$ and $T$, a function $f$ from $S$ to $T$ is a rule which assigns each $s \in S$ exactly one element in $T$. This element is denoted $f(s)$. We often write $f$ as $f: S \rightarrow T$ to make clear the choice of $S$ and $T$. 
Definition 1.10. The union of two sets $S$ and $T$, denoted $S \cup T$, is the set consisting of all elements in either $S$ or $T$.

Example 1.11. If $S=\{A, B\}$ and $T=\{B, C\}$, then $S \cup T=\{A, B, C\}$.

Definition 1.12 (Subset). For sets $S$ and $T$, we say $S$ is a subset of $T$, and write $S \subset T$, if every element of $S$ is also an element of $T$.

Example 1.13. We sometimes specify a subset via a property its elements satisfy. For example, consider the set

$$
S=\{0,1,2,3,4,5,6,7,8,9\}
$$

and the subset

$$
T=\{0,2,4,6,8\}
$$

i.e., $S$ denotes the set of all digits, and $T$ denotes the set of all even digits. Then $T$ can be specified as the set of integers $z$ such that $z$ is divisible by 2 . We write this in symbols as follows:

$$
T=\{z \in S \mid z \text { is divisible by } 2\} .
$$

the symbol "|" translates into words as "such that."

Definition 1.14 (Complement). If $S \subset T$, then $T \backslash S$, called the complement of $S$ in $T$, is the set of elements in $T$ which are not also in $S$. That is,

$$
T \backslash S=\{t \in T \mid t \notin S\} .
$$

Example 1.15. If $T=\{A, B, C\}$ and $S=\{A\}$, then $T \backslash S=\{B, C\}$.

Definition 1.16 (Cartesian Product). For sets $S$ and $T$, the Cartesian Product of $S$ and $T$, denoted $S \times T$, is the set of all ordered pairs $(s, t)$ with $s \in S$ and $t \in T$. In symbols, we write this as follows:

$$
S \times T=\{(s, t) \mid s \in S, t \in T\} .
$$

Example 1.17. For $S=\{1,2\}$ and $T=\{A, B\}$,

$$
S \times T=\{(1, A),(1, B),(2, A),(2, B)\} .
$$

Example 1.18. By definition, $\mathbb{R} \times \mathbb{R}$ is the set of all ordered pairs of real numbers. $\mathbb{R} \times \mathbb{R}$ is usually written as $\mathbb{R}^{2}$.

Definition 1.19 ( $n$-Fold Cartesian Product). More generally, we can define the Cartesian product

$$
S_{1} \times S_{2} \times \cdots \times S_{n}
$$

of $n \geq 1$ sets $S_{1}, S_{2}, \ldots, S_{n}$ to be the set of all ordered lists $\left(s_{1}, s_{2}, \ldots, s_{n}\right)$ of length $n$, where $s_{i} \in S_{i}$ for each $i$. Thus, in symbols, the definition is:

$$
S_{1} \times S_{2} \times \cdots \times S_{n}=\left\{\left(s_{1}, s_{2}, \ldots, s_{n}\right) \mid s_{i} \in S_{i}\right\} .
$$

Example 1.20. As a set, $n$-dimensional Euclidean space $\mathbb{R}^{n}$ is the Cartesian product of $n$ copies of the real numbers $\mathbb{R}$. 
We often write

$$
S_{1} \times S_{2} \times \cdots \times S_{n}
$$

in more concise form as $\prod_{i=1}^{n} S_{i}$. Along similar lines, if $V=\left\{v_{1}, v_{2}, \ldots, v_{n}\right\}$ is a finite ordered set and we have a set $S_{v}$ for each $v \in V$, then we sometimes write

$$
S_{v_{1}} \times S_{v_{2}} \times \cdots \times S_{v_{n}}
$$

as $\prod_{v \in V} S_{v}$.

Definition 1.21 (Coordinate Projection). Given $W \subset V$, there is a natural function

$$
\rho: \prod_{v \in V} S_{v} \rightarrow \prod_{v \in W} S_{v}
$$

which retains all elements indexed by $W$, while discarding all elements indexed by $V \backslash W$. We call this function a co-ordinate projection.

Example 1.22. If $V=\{1,2,3\}$ and $W=\{1,3\}$, then for $(a, b, c) \in \prod_{v \in V} S_{v}$, we have that $\rho(a, b, c)=$ $(a, c)$.

Definition 1.23 (Partition). A partition $P$ of a set $S$ is a set such that:

- Elements of $P$ are non-empty subsets of $S$.

- Each element of $S$ is contained in exactly one element of $P$.

Example 1.24. $\{\{1\},\{2,3\}\}$ is a partition of $\{1,2,3\} .\{\{1,2,3\}\}$ is also a partition of $\{1,2,3\}$.

\subsection{Graph Theory Preliminaries}

Definition 1.25 (Graph). A (finite directed) graph $G$ consists of a finite set $V$, called the vertices, together with a subset $E$ of $V \times V$ called the edges. We will often write $\left(v_{1}, v_{2}\right) \in E$ as $\left[v_{1}, v_{2}\right]$. If $e=\left[v_{1}, v_{2}\right] \in E$, then we say $e$ is incident to $v_{1}$ and $v_{2}$. Symmetrically, we also say that $v_{1}$ and $v_{2}$ are incident to $e$.

Definition 1.26 (Bipartite Graph). A graph $G$ is said to be bipartite if a partition $V=A \cup B$ of the vertices $V$ is specified such that for each edge $[v, w] \in E$, that $v \in A$ and $w \in B$.

Definition 1.27. A matching $M$ in a graph $G$ is a subset of the edges $E$ such that each vertex of $G$ is incident to at most one edge in $M$.

\section{Multitasking Environments}

In modeling multitask performance, it will be convenient to restrict attention to a small subset of multitasks that we may wish to ask an agent to perform. In this section, we introduce the relevant definitions. We then give examples in the context of the well known Stroop task (Stroop, 1935), and an extended version of this task described introduced by Feng et al. (2014). 
Definition 2.1 (Task). A task is any function $f: S \rightarrow R$. We call $S$ the stimulus set, and $R$ the response set. ${ }^{1}$

Definition 2.2 (Multitasking Environment). A (multitasking) environment $\mathcal{X}$ consists of:

1. A finite bipartite graph $\mathcal{G}$ with vertex set partition $V=V_{S} \cup V_{R}$ and edge set $E$.

2. Two collections of ordered sets

$$
S=\left\{S_{i}\right\}_{i \in V_{S}} \text { and } R=\left\{R_{j}\right\}_{j \in V_{R}}
$$

3. For each $[i, j] \in E$, a task $f_{[i, j]}: S_{i} \rightarrow R_{j}$; we let $T$ denote the collection of these tasks.

Remark 2.3. The requirement that $V_{S}$ and $V_{R}$ are ordered is just an expository convenience; the specific choice of order on these sets is not important.

Definition 2.4 (Multitask). A multitask (in $\mathcal{X}$ ) is a subset of $T$ of the form

$$
f_{M}:=\left\{f_{[i, j]} \mid[i, j] \in M\right\}
$$

for some matching $M \subset E$. Thus, a multitask consists of a set of tasks in the environment $\mathcal{X}$ that involve separate stimulus sets and response sets. We call the number of edges in $M$ the size of $f_{M}$.

This definition of multitask is in accord with previous treatments of multitasking in the psychological literature (Feng et al., 2014; Navon \& Gopher, 1979; Meyer \& Kieras, 1997; Musslick et al., 2016; Pashler, 1994; Townsend \& Wenger, 2004a; Welford, 1952; Wickens, 1991).

We let $\mathcal{M}$ denote the set of all multitasks in $\mathcal{X}$.

For $M \subset E$ a matching, let

$$
\begin{aligned}
& V_{S}^{M}:=\left\{i \in V_{S} \mid \exists j \in V_{R} \text { with }[i, j] \in M\right\}, \\
& V_{R}^{M}:=\left\{j \in V_{R} \mid \exists i \in V_{S} \text { with }[i, j] \in M\right\} .
\end{aligned}
$$

Thus, $V_{S}^{M}$ is the set of vertices in $V_{S}$ which are incident to an edge in the matching $M$, and $V_{R}^{M}$ has an analogous interpretation. $V_{S}^{M}$ and $V_{R}^{M}$ inherit orders from the respective orders on $V_{S}$ and $V_{R}$.

$$
\begin{aligned}
& S_{M}=\prod_{i \in V_{S}^{M}} S_{i}, \\
& R_{M}=\prod_{j \in V_{R}^{M}} R_{j} .
\end{aligned}
$$

Note that, ordering the edges of $M$ to be consistent with the order on $V_{M}^{S}$, we can think of $f_{M}$ as a single function

$$
f_{M}: S_{M} \rightarrow R_{M}
$$

given by

$$
f_{M}\left(s_{1}, \ldots, s_{k}\right)=\left(f_{1}\left(s_{1}\right), \ldots, f_{k}\left(s_{k}\right)\right) .
$$

\footnotetext{
${ }^{1}$ This is quite an abstract definition of task; of course, not every mathematical function is likely to have a cognitively meaningful interpretation as a task. But we propose that every cognitively meaningful task can be modeled as a mathematical function.
} 
Remark 2.5. When considering a Cartesian product $\prod S_{i}$ of stimuli sets it is common in the psychological literature to refer to the individual factors $S_{i}$ as "stimulus dimensions". However, we will avoid this language, and simply refer to these as factors as stimulus sets. We follow the analogous convention for products of response sets.

Remark 2.6. Note that Definition 2.4 does not allow two of the individual functions in a multitask to share a stimulus set $S_{i}$. This constraint is well suited to the sorts of psychological settings we are most interested in modeling, and simplifies the setup of certain quantitative analyses. However, our formalism could be modified to allow the individual functions in a multitask to share stimulus sets.

Example 2.7 (Stroop Task). We consider the Stroop paradigm (Stroop, 1935) in which participants are prompted with color words (e.g. "RED" or "GREEN") displayed in a particular color ink (e.g. red ink or green ink). In one condition, participants are tasked with naming the ink color of the stimulus while ignoring the word (color naming). Alternatively, participants may be tasked with reading the word out loud while ignoring the color (word reading). Critically, in some conditions the color of the ink and the word are the same (congruent conditions), whereas in other conditions they are different (incongruent conditions).

The task environment of the Stroop paradigm is illustrated in Fig. 1. To formally define the environment, we take $V_{S}=\{1,2\}, V_{R}=\{a\}, E=\{1 a, 2 a\}$,

$$
\begin{aligned}
& S_{1}=\{\text { red [text color], green [text color] }\}, \\
& S_{2}=\{\text { RED [text word], GREEN [text word] }\},
\end{aligned}
$$

and $R=\left\{R_{a}\right\}$, where

$$
R_{a}=\{\text { say "red", say "green" }\} \text {. }
$$

Here, we are writing an edge $[i, j]$ simply as $i j$ to simplify notation. The functions $f_{1 a}$ and $f_{2 a}$ model the color naming and word reading tasks, respectively.

Example 2.8 (Extended Stroop Task). Previous work has proposed a dual-tasking extension of the Stroop paradigm (Feng et al., 2014). In this extended Stroop paradigm, participants are presented with color words (e.g. "RED" or "GREEN") displayed in colored ink (red or green) on a specified location on the screen (left or right). The tasks in this paradigm require the participant to respond either verbally or via a button press on a keyboard. In addition to the color naming and word reading tasks of the original Stroop paradigm, participants have to perform two more tasks: (1) location pointing, wherein participants are asked to indicate the location of the stimulus with a button press (e.g. press left button if the word is presented on the left); and (2) word pointing, wherein participants map words onto button presses (e.g. press the left button if the word "RED" appears).

The task environment of the extended Stroop paradigm is illustrated in Fig. 2. To formally define the environment, we take $V_{S}=\{1,2,3\}, V_{R}=\{a, b\}, E=\{1 a, 2 a, 2 b, 3 b\}$,

$$
\begin{aligned}
& S_{1}=\{\text { red [text color], green [text color] }\}, \\
& S_{2}=\{\text { RED [text word], GREEN [text word] }\}, \\
& S_{3}=\{\text { left [location], right [location] }\},
\end{aligned}
$$




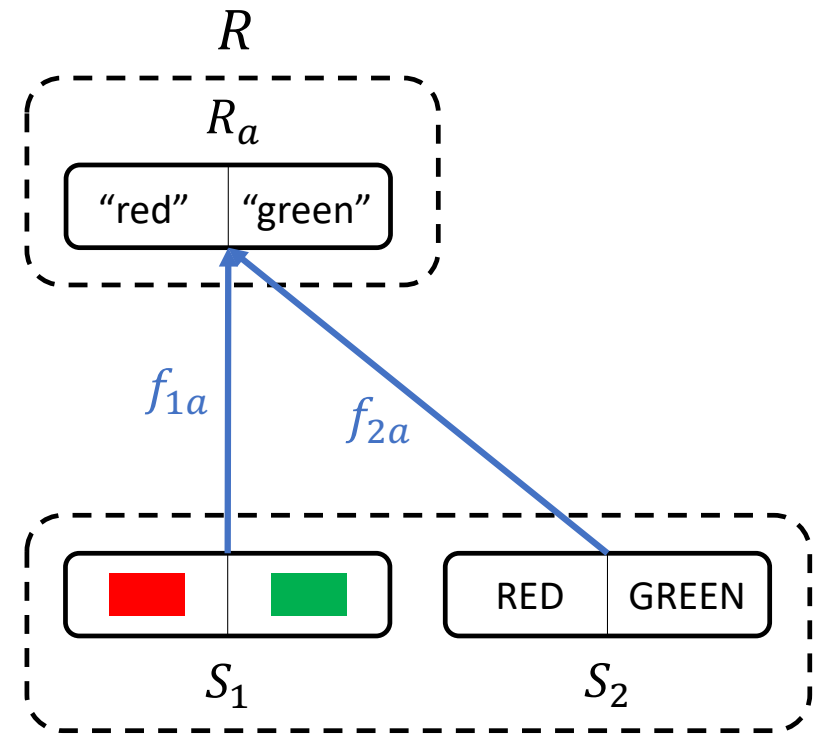

$S$

Figure 1: Environment of the Stroop task.

and $R=\left\{R_{a}, R_{b}\right\}$, where

$$
\begin{aligned}
& R_{a}=\{\text { say "red", say "green" }\} \\
& R_{b}=\{\text { press left button, press right button }\}
\end{aligned}
$$

Tasks $f_{1 a}$ and $f_{2 a}$ correspond to color naming and word reading, as in Example 2.7; $f_{2 b}$ and $f_{3 b}$ model the word pointing and location pointing tasks, respectively.

In addition to single tasks, participants' performance is measured on two different multitasks: In multitask $f_{\{1 a, 3 b\}}$, participants are asked to simultaneously perform color naming and location pointing; in multitask $f_{\{1 a, 2 b\}}$, participants are asked to perform color naming and word pointing simultaneously.

\section{Agents}

We next model the agent (e.g., human or artificial neural network) which attempts to perform a multitask in a given environment $\mathcal{X}$.

Definition 3.1. An agent $\mathcal{A}$ (in $\mathcal{X}$ ) consists of:

1. An augmented response set $R_{i}^{\prime}$ containing $R_{i}$ as a subset, for each $i \in V_{R}$.

2. For each $M \in \mathcal{M}$, a choice of (possibly stochastic) function

$$
\mathcal{A}_{M}: \prod S \rightarrow \prod R^{\prime}
$$

where $\prod S=\prod_{i \in V_{S}} S_{i}$ and $\prod R^{\prime}=\prod_{i \in V_{R}} R_{i}^{\prime}$. (That is, $\prod S$ and $\prod R^{\prime}$ are the Cartesian products of all stimuli sets and augmented response sets in the environment, respectively.) 


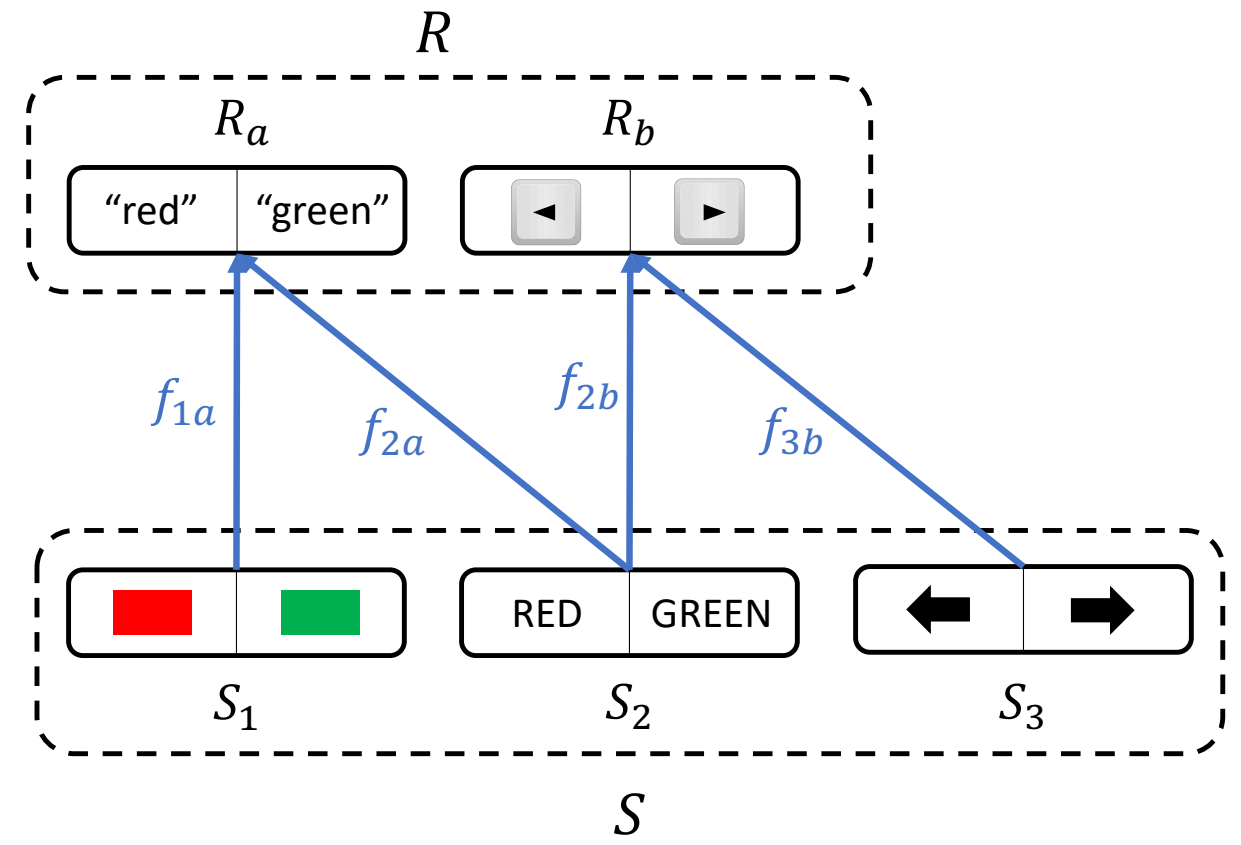

Figure 2: Environment of the extended Stroop task. $f_{M}$.

Intuitively, we think of $\mathcal{A}_{M}$ as a specification of how the agent attempts to perform the multitask

This definition of an agent requires further explanation:

- We take the domain of $\mathcal{A}_{M}$ to be $\prod S$ (rather than $S_{M}$ ) because we wish to model the situation where the agent is always presented with a stimulus from every stimulus set. This modeling assumption is important, e.g., in modeling the Stroop task; see Section 4.1 below.

- We take the codomain of $\mathcal{A}_{M}$ to be a product indexed by all of $V_{R}$ (rather than by $V_{R}^{M}$ ) because the agent may exhibit cognitively interesting behavior in irrelevant response dimensions, even though this does not contribute to successful completion of the task. We may wish to model such behavior.

- Moreover, we take the codomain of $\mathcal{A}_{M}$ to be $\prod R^{\prime}$ (rather than $\prod_{i \in V_{R}} R_{i}$ ) because the agent's set of responses for each stimulus dimension may not be limited to the set of task-relevant responses.

Remark 3.2. For the examples we consider later in this note, it will suffice to assume that $R_{i}^{\prime}$ has at most one element which is not in $R_{i}$. We interpret the extra element of $R_{i}^{\prime}$ to be a generic "other response".

Definition 3.3 (Success Rate). If we assume that $\prod S$ is endowed with a probability distribution (e.g., if $\prod S$ is finite and is given the uniform distribution), then we may define the success rate of $\mathcal{A}$ on a multitask $f_{M}$ in $\mathcal{X}$ by

$$
P\left(\rho_{M}\left(\mathcal{A}_{M}(s)\right)=f_{M}(s)\right)
$$


where

$$
\rho_{M}: \prod R^{\prime} \rightarrow \prod_{i \in V_{R}^{M}} R_{i}^{\prime}
$$

is the coordinate projection, i.e., the function which simply discards responses irrelevant to the multitask $M$ (see Definition 1.21). Intuitively, this is the probability that the agent performs the multitask correctly.

Remark 3.4. In practice, the response sets in the environment may come equipped with distances, i.e., ways of measuring how close two responses are to each other, and it may be of interest to quantify how close an agent's performance on a multitask is to the correct response. However, our definition of success rate does not take distances into account. Thus, this notion of success rate is suitable for discrete (i.e., categorical) responses, as we have in the Stroop task and extended Stroop task, but it is not suitable for continuous responses. That said, it would not be difficult to adapt the definition of success rate to give a similar measure which takes into account distance.

Definition 3.5 (Perfect Agent). We say that an agent $\mathcal{A}$ in $\mathcal{X}$ is perfect if for all multitasks $M$ in $\mathcal{X}$, the success rate of $\mathcal{A}$ on $M$ is 1 . That is, a perfect agent is one which performs every multitask perfectly with probability 1 .

Note that a perfect agent always exists, but need not be unique, for instance because an agent's behavior in irrelevant response dimensions does not affect the success rate.

\section{Examples of Agents}

The recent literature on multitasking has introduced several natural models of agents. We now review two of these and show how they can be described in terms of the formal language of the previous section.

\subsection{Naive Bipartite Agents}

We first introduce a particularly simple deterministic model which is completely specified by the multitasking environment, without having to choose any additional parameters. We call this model the naive bipartite agent. This model implicitly incorporates the assumption that to perform a task, the agent exercises cognitive control by activating internal representations of the stimuli and response sets relevant to that task. It is assumed that cognitive control can directly activate only such representations, and not the specific connections between them.

This model is a special case of an agent considered in Musslick et al. (2016) and Alon et al. (2017); an antecedent of the general construction appears in Feng et al. (2014). We study the fully general version below, in Section 4.2.

Definition 4.1 (Compromised Edge). Let $G$ be a bipartite graph with vertex set partition $V=A \cup B$ and edge set $E$. Let $M$ be a matching in $G$. We say an edge $m \in M$ is compromised in $M$ if there exists an edge $m^{\prime} \in M$ not equal to $m$, and an edge $e \in E$ such that

- $m^{\prime}$ and $e$ share a vertex in $A$, and

- $m$ and $e$ share a vertex in $B$.

Fig. 3 illustrates a compromised edge. 


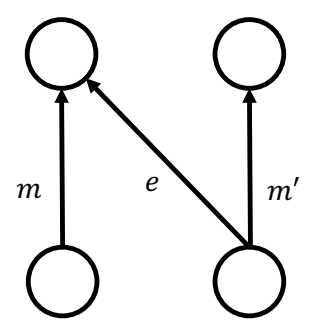

Figure 3: Example of a compromised edge $m$ in a bipartite graph.

Definition 4.2 (Naive Bipartite Agent). To define the naive bipartite agent $\mathcal{A}$, for $j \in V_{R}$, we define the augmented response sets as $R_{j}^{\prime}=R_{j} \cup\left\{\right.$ null $\left._{j}\right\}$, where null , $_{j} \notin R_{j}$ represents a task-irrelevant response (see Remark 3.2).

$$
\mathcal{A}_{M}\left(s_{1}, \ldots, s_{\left|V_{S}\right|}\right)=\left(r_{1}, \ldots, r_{\left|V_{R}\right|}\right)
$$

where

$$
r_{j}= \begin{cases}f_{[i, j]}\left(s_{i}\right) & \text { if there exists an edge }[i, j] \in M \text { with }[i, j] \text { not compromised in } M, \\ \text { null }_{j} & \text { otherwise. }\end{cases}
$$

Thus, when attempting to perform a multitask $f_{M}$, the bipartite agent performs an individual task $f_{m}$ correctly if and only if the edge $m$ is not compromised.

Intuitively, if $m$ is compromised in $M$, with $m^{\prime}=\left[i^{\prime}, j^{\prime}\right]$ and $e$ as above, then when attempting to perform multi-task $f_{M}$, the bipartite agent must activate the stimulus set $S_{i^{\prime}}$ in order to simultaneously attempt task $f_{m^{\prime}}$. But since the agent is only capable of turning on and off inputs and outputs, and cannot otherwise control the specific connections between them, the agent's representation of $f_{e}$ then interferes with its attempt at $f_{m}$, causing $f_{m}$ to be performed incorrectly.

Note that the success rate of the bipartite agent $\mathcal{A}$ on multitask $f_{M}$ is 1 if and only if no edge in $f_{M}$ is compromised.

Remark 4.3. In previous work (Musslick et al., 2016; Alon et al., 2017), the maximum number of tasks that a bipartite agent can perform without performance loss was defined as the size of the largest induced matching $M \in \mathcal{M}^{2}$. This is equivalent to the size of the largest matching $M \in \mathcal{M}$ in which no edge is compromised, that is, for which the success rate is equal to 1.

Performance on the Stoop Task. To name the color of a Stroop stimulus, the agent must exercise control, by activating the vertex representing colors in the input layer and the vertex representing verbal responses in the output layer (Fig. 4A). The edge indexing the color naming task forms a matching in $E$, and is compromised by no other edge. Thus, the agent can name the color of a Stroop stimulus with a success rate of 1 .

Performance on the Extended Stoop Task. We next consider performance of the bipartite agent in the extended Stroop environment (Fig. 4B). In the first multitasking condition, the agent is asked to multitask color naming with location pointing. Note that the two tasks form a multitask in $\mathcal{X}$ (cf., $f_{1 a}, f_{3 b}$ in Fig. 2). Executing the two tasks requires the control system to turn on the

\footnotetext{
${ }^{2}$ This corresponds to the size of the maximum independent set of the squared line graph of the bipartite agent.
} 

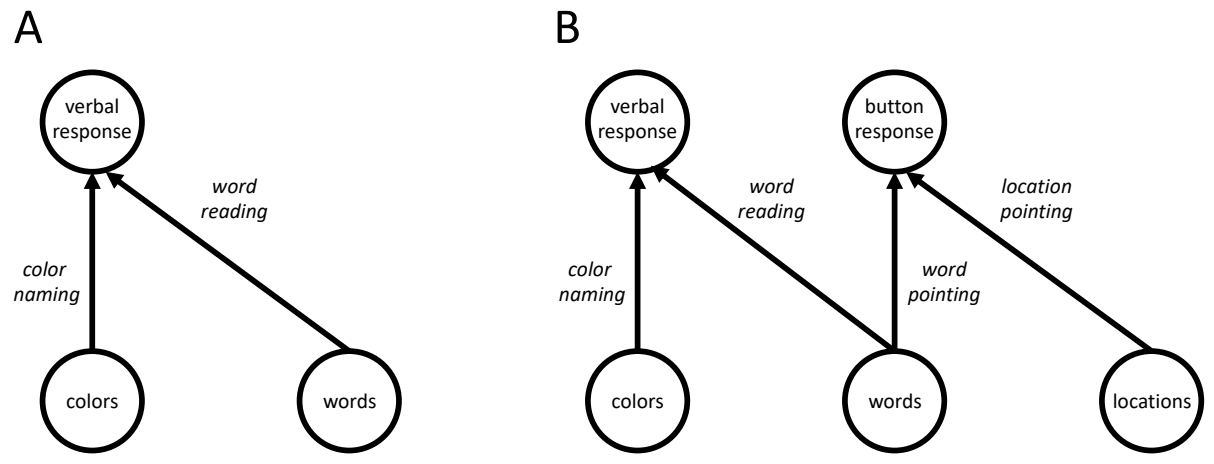

Figure 4: Example of the Naive Bipartite Agent for (A) the Stroop environment and (B) the extended Stroop environment.

vertices representing colors and locations in the input layer, as well as verbal responses and button responses in the output layer. There exists no edge that compromises either the edge representing the color naming task or the edge representing the location pointing task. Thus, the agent can multitask the two tasks with a success rate of 1 .

In the second multitasking condition, the agent is asked to multitask color naming with word pointing. Similar to the first multitasking condition, the two tasks form a multitask in $\mathcal{X}$ (cf., $f_{1 a}, f_{2 b}$ in Fig. 2). When asked to multitask the two tasks, the control system has to turn on the vertices representing colors and words in the input layer, as well as the vertices representing verbal responses and button responses in the output layer of the bipartite graph. This control policy, however, implicitly engages a third task that connects the input vertex for words and the output vertex for verbal responses (word reading), compromising the edge representing the color naming task. Thus, the success rate of multitasking color naming and word pointing corresponds to 0 .

\subsection{Generalized Bipartite Agents}

Above, we have seen that the naive bipartite agent performs poorly on the extended Stroop task. In general, multitasking capabilities of the naive bipartite agent are very limited in a way that differs from humans, who can sometimes multitask very well, even for multitasks involving tasks indexed by compromised edges.

This motivates consideration of an extension of the naive bipartite agent model which is capable of expressing a broader range of multitasking capabilities. This extension, which we call the generalized bipartite agent, appears in Musslick et al. (2016); Alon et al. (2017).

The key idea is that a generalized bipartite agent may maintain multiple, intermediate representations of the same stimulus set, with different representations used to perform different tasks. Like the naive bipartite model, the generalized model implicitly incorporates the assumption that to execute a task, it is required to exercise control by activating the representations relevant to that task. Furthermore, control may act only on the representations of stimuli and responses, and not on the connections between them.

To specify a generalized bipartite agent in the environment $\mathcal{X}$, we will use notation for environments of Definition 2.2. In particular, we call the bipartite graph underlying the environment $\mathcal{G}$, its vertices $V=V_{S} \cup V_{R}$, and its edges $E$.

Definition 4.4 (Derived Bipartite Graph). For each vertex $v \in V_{S}$, let $E_{v}$ denote the set of edges in 
$E$ incident to $v$. To specify a generalized bipartite agent in $\mathcal{X}$, we first fix a choice of partition $\Gamma^{v}$ of $E_{v}$, for each $v \in V_{S}$. We let

$$
\Gamma:=\bigcup_{v \in V_{s}} \Gamma^{v}
$$

i.e., $\Gamma$ is the union of all of these partitions. We then specify a second bipartite graph $\mathcal{G}^{\Gamma}$ with vertex set partition $V^{\Gamma}=\Gamma \cup V_{R}$ and edge set $E^{\Gamma}$ defined in the following way (see Fig. 5 for an example): for

$$
v^{\prime}=\left\{\left[v, w_{1}\right],\left[v, w_{2}\right], \ldots,\left[v, w_{n}\right]\right\} \in \Gamma,
$$

the edges incident to $v^{\prime}$ in $E^{\Gamma}$ are

$$
\left[v^{\prime}, w_{1}\right],\left[v^{\prime}, w_{2}\right], \ldots,\left[v^{\prime}, w_{n}\right] .
$$

Note that there is a 1-1 correspondence between $E$ and $E^{\Gamma}$. By slight abuse of notation, for each edge $e \in E$, we also denote its corresponding edge in $E^{\Gamma}$ by $e$. Note further that under the correspondence between $E$ and $E^{\Gamma}$, a matching $M$ in $G$ yields a matching $M^{\Gamma}$ in $G^{\Gamma}$. However, the converse is not always true.

Definition 4.5 (Generalized Bipartite Agent). In close analogy to Definition 4.2, we now define the generalized bipartite agent $\mathcal{A}^{\Gamma}$ as follows: We take the augmented response sets to be $R_{j}^{\prime}=$ $R_{j} \cup\left\{\right.$ null $\left._{j}\right\}$, where null $_{j} \notin R_{j}$ represents a task-irrelevant response, exactly as in Definition 4.2. For $M$ a matching in $\mathcal{G}$, we take

$$
\mathcal{A}_{M}^{\Gamma}\left(s_{1}, \ldots, s_{\left|V_{S}\right|}\right)=\left(r_{1}, \ldots, r_{\left|V_{R}\right|}\right),
$$

where

$$
r_{j}= \begin{cases}f_{[i, j]}\left(s_{i}\right) & \text { if there exists an edge }[i, j] \in M \text { with }[i, j] \text { not compromised in } M^{\Gamma}, \\ \text { uull }_{j} & \text { otherwise. }\end{cases}
$$

Remark 4.6 (Interpretation of the generalized bipartite agent). When $\Gamma^{v}=\left\{E_{v}\right\}$ for each $v \in V_{S}$ (i.e., each partition $\Gamma^{v}$ consists of just a single set containing all edges in $E$ incident to $v$ ), the generalized bipartite agent is the same as the naive bipartite agent. In prior work, this agent is said to have a minimal basis set representation of the stimuli set. For this agent, each set $\Gamma^{v}$ has as few elements as possible. At the opposite extreme, we can take $\Gamma=E$ (i.e., each element of the partition $\Gamma^{v}$ consists of just a single edge in $E$.) In prior work, this agent is said to have a tensor product representation of the stimuli set. In this case, we interpret the generalized bipartite agent as maintaining a distinct representation of the stimulus set $S_{v}$ for each edge in $G$ incident to $V$. Such an agent will have success rate 1 on every multitask. In between these two extremes, a generalized bipartite agent also can represent scenarios where the internal representation of a stimulus is neither fully shared nor fully separated. This is illustrated in Fig. 5.

Performance on the Stoop Task. To illustrate performance of the generalized bipartite agent, we consider two instances of such an agent shown in Fig. 6. To name the color of a Stroop stimulus, both agents must exercise control, by activating the vertex representing colors in the input layer and the vertex representing verbal responses in the output layer. For both agents, the color naming task forms a matching in $E$, and is compromised by no other edge. Thus, both agents can name the color of a Stroop stimulus with a success rate of 1 . 
A

Graph Underlying the Task Environment $\mathcal{X}$

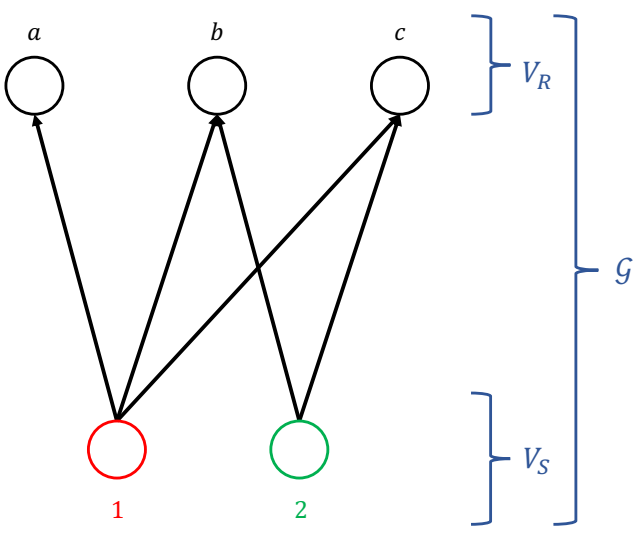

B

Graph Specifying the Generalized Bipartite Agent $A^{\Gamma}$

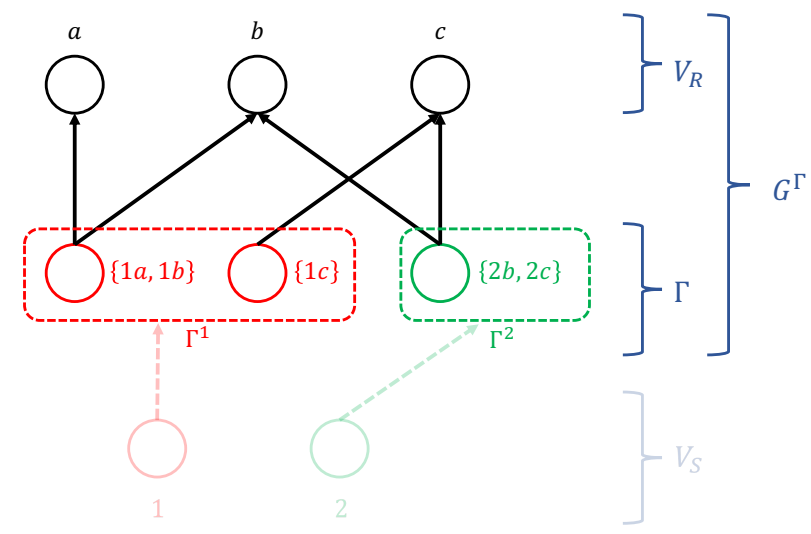

Figure 5: Example of a task environment with a generalized bipartite agent. (A) The graph $\mathcal{G}$ underlying the environment $\mathcal{X}$ has vertex set $V_{S} \in\{1,2\}$ representing the stimuli, and vertex set $V_{R} \in\{a, b, c\}$ representing the responses. The stimuli vertices 1 and 2 are depicted in red and green, respectively. (B) The generalized bipartite agent is specified by the derived bipartite graph $G^{\Gamma}$. To define $G^{\Gamma}$, we partition the edges incident to vertex 1 into two sets $\{1 a, 2 b\},\{1 c\}$, and partition the edges incident to vertex 2 into a single set $\{2 a, 2 b, 2 c\}$. (For brevity of notation, an edge $[i, j]$ is represented as $i j)$. Correspondingly, vertex 1 is split into two vertices $\{1 a, 1 b\},\{1 c\}$ and vertex 2 is mapped to a single vertex $\{2 b, 2 c\}$. The set of these three vertices is denoted $\Gamma$, and describes the agent's internal representation of the stimulus sets in the environment $\mathcal{X}$. Observe that the matching $M=\{1 a, 2 c\}$ in $\mathcal{G}$ also provides a matching $M^{\Gamma}$ in $G^{\Gamma}$. The naive bipartite agent described in Definition 4.2 cannot perform the multitask corresponding to $M$ since $2 c$ is compromised by $1 c$ in $M$. However, the generalized bipartite agent depicted here can execute this multitask with a success rate of 1 because neither $1 a$, nor $2 c$ is compromised in $M^{\Gamma}$.

A

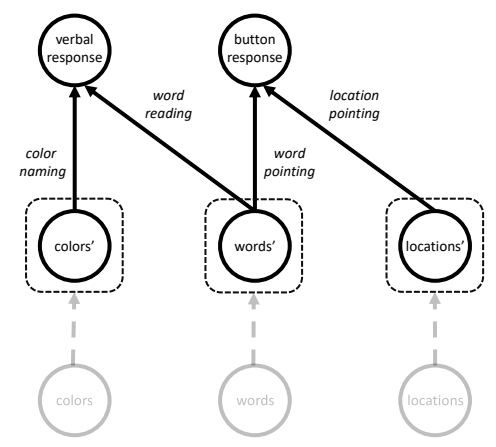

$\mathrm{B}$

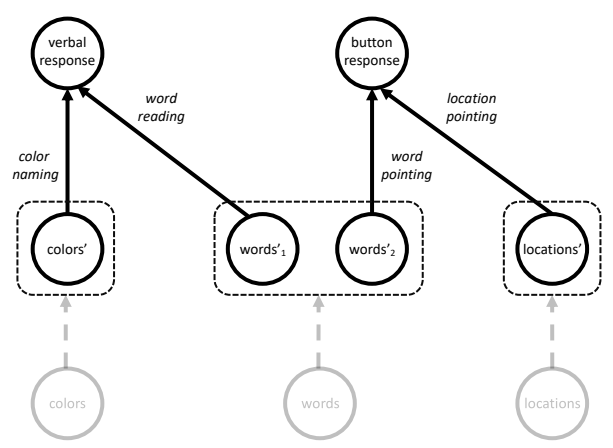

Figure 6: Two instances of the Generalized Bipartite Agent for the extended Stroop environment. 
Performance on the Extended Stoop Task. In the first multitasking condition, both agents are asked to multitask color naming with location pointing. Observe that the two tasks form a multitask in $\mathcal{X}$, i.e. a matching in $\mathcal{G}$, as well as in $G^{\Gamma}$, for both agents. Executing both tasks requires both agents to exercise control, by engaging the input nodes for colors and locations. For both agents, there exists no edge that compromises either the edge representing the color naming task or the edge representing the location pointing task. Thus, both agents can multitask the two tasks with a success rate of 1 .

In the second multitasking condition, both agents are asked to multitask color naming with word pointing. Again, the two tasks form a multitask in $\mathcal{X}$ for both agents. When asked to multitask the two tasks, the control system has to turn on the vertices representing verbal responses and button responses in the output layer, as well as the vertices representing colors and words in the input layer that are incident to the edges representing the color naming and word pointing task, respectively. However, note that the two agents shown in Fig. 6 have different partitions of the edges incident to the word input. Whereas the first agent shown in Fig. 6A maps the word input to a single vertex words', the second agent shown in Fig. 6B maps the word input to two vertices $w o r d s_{1}^{\prime}$, words $s_{2}^{\prime}$. In the first agent, the control policy implicitly engages a third task that connects the input vertex for words (words') and the output vertex for naming (word reading), compromising the edge representing the color naming task. Thus, the success rate of multitasking color naming and word pointing corresponds to 0 for the agent shown in Fig. 6A. However, in the second agent, the edge representing the color naming task is not compromised by the word reading task, because engaging the word representation for word pointing (word $s_{2}^{\prime}$ ) does not lead to an implicit activation of the representation for word reading ( $\left.w o r d s_{1}^{\prime}\right)$. Thus, the agent shown in Fig. 6B is can execute the second multitask with a success rate of 1.

\section{Conclusion}

We have introduced a formal language for the study of multitasking behavior. The proposed formalism entails a definition of the task environment (which in particular specifies the multitasks an agent might perform), as well as a definition of the multitasking agent. We have illustrated the definition of a task environment on variants of the Stroop task (Stroop, 1935; Feng et al., 2014), and shown how simple agents perform on these variants (Feng et al., 2014; Musslick et al., 2016). We hope that our formalism will facilitate quantitative efforts to explain multitasking phenomena. In future work, we aim to use our framework to study other multitasking agents that have been proposed in the literature, such as neural network models (Musslick et al., 2017; Musslick \& Cohen, 2019) and parallel processing channels (Townsend \& Wenger, 2004b; Townsend \& Altieri, 2012), as well as novel, weighted bipartite agents.

\section{References}

Alon, N., Reichman, D., Shinkar, I., Wagner, T., Musslick, S., Cohen, J. D., .. others (2017). A graph-theoretic approach to multitasking. In Advances in neural information processing systems (pp. 2100-2109).

Feng, S. F., Schwemmer, M., Gershman, S. J., \& Cohen, J. D. (2014). Multitasking vs. multiplexing: toward a normative account of limitations in the simultaneous execution of controldemanding behaviors. Cognitive, Affective, \& Behavioral Neuroscience, 14(1), 129-146.

Logan, G. D., \& Gordon, R. D. (2001). Executive control of visual attention in dual-task situations. Psychological review, 108(2), 393. 
Meyer, D. E., \& Kieras, D. E. (1997). A computational theory of executive cognitive processes and multiple-task performance: Part I. Basic mechanisms. Psychological review, 104(1), 3.

Musslick, S., \& Cohen, J. D. (2019). A mechanistic account of constraints on control-dependent processing: Shared representation, conflict and persistence. In Proceedings of the 41st Annual Meeting of the Cognitive Science Society (pp. 849-855). Montreal, CA.

Musslick, S., Dey, B., Özcimder, K., Patwary, M. M. A., Willke, T. L., \& Cohen, J. D. (2016). Controlled vs. automatic processing: A graph-theoretic approach to the analysis of serial vs. parallel processing in neural network architectures. In Proceedings of the 38th Annual Meeting of the Cognitive Science Society (p. 1547-1552).

Musslick, S., Saxe, A., Özcimder, K., Dey, B., Henselman, G., \& Cohen, J. D. (2017, August). Multitasking capability versus learning efficiency in neural network architectures. In Proceedings of the 39th Annual Meeting of the Cognitive Science Society (p. 829-834).

Navon, D., \& Gopher, D. (1979). On the economy of the human-processing system. Psychological Review, 86(3), 214.

Pashler, H. (1994). Dual-task interference in simple tasks: data and theory. Psychological bulletin, 116(2), 220.

Stroop, J. R. (1935). Studies of interference in serial verbal reactions. Journal of experimental psychology, 18(6), 643.

Townsend, J. T., \& Altieri, N. (2012). An accuracy-response time capacity assessment function that measures performance against standard parallel predictions. Psychological review, 119(3), 500 .

Townsend, J. T., \& Wenger, M. J. (2004a). The serial-parallel dilemma: A case study in a linkage of theory and method. Psychonomic Bulletin E Review, 11(3), 391-418.

Townsend, J. T., \& Wenger, M. J. (2004b). A theory of interactive parallel processing: new capacity measures and predictions for a response time inequality series. Psychological review, 111(4), 1003.

Welford, A. T. (1952). The psychological refractory period and the timing of high-speed performance-a review and a theory. British Journal of Psychology, 43(1), 2.

Wickens, C. D. (1991). Processing resources and attention. Multiple-task performance, 1991, 3-34. 